\title{
Suzaku observations of the radio galaxy Fornax A west lobe
}

\author{
Hiromi Seta ${ }^{1}$, Makoto S. Tashiro ${ }^{1}$ and Naoki Isobe ${ }^{2}$ \\ ${ }^{1}$ Department of Physics, Saitama University, 255 Shimo-Okubo, Sakura, Saitama, 338-8570, \\ Japan \\ email: seta@heal.phy.saitama-u.ac.jp \\ ${ }^{2}$ Department of Astronomy, Kyoto University, Kitashirakawa-Oiwake-cho, Skyo-ku, Kyoto \\ 606-8502, Japan
}

\begin{abstract}
We performed mapping observations of the Fornax A west lobe with Suzaku in order to measure X-ray brightness distribution. Thanks to the low and stable background of Suzaku, we succeeded in detecting the faint diffuse X-ray emission from the west lobe. Performing careful corrections to the obtained images, we finally measured the X-ray brightness profile extending over the lobe. By comparing the X-ray and radio profiles, the magnetic field found to be fairly constant at $\sim 1 \mu \mathrm{G}$ over the lobe, while the electron energy distribution is suggested to concentrate on the lobe center.
\end{abstract}

Keywords. galaxies: individual: Fornax A, radiation mechanisms: non-thermal

\section{Introduction}

In the last several years, detections of cosmic microwave background boosted inverses Compton (IC) X-ray emission have been successively reported from radio lobes (e.g., Isobe et al. 2002). Using the X-ray and radio fluxes, we are able to estimate physical parameters such as the electron and magnetic energy densities, $u_{e}, u_{m}$, and the magnetic field strength $B$ in the lobes.

In this paper, we evaluate spatial distributions of these physical parameters precisely by means of obtaining a detailed IC X-ray brightness distribution, which has ever assumed to have the same spatial distribution of radio emission. Fornax $\mathrm{A}$ is one of the brightest and largest radio galaxies. The hard X-ray up to $20 \mathrm{keV}$ was detected for the first time from the west lobe with Suzaku, which showed that the Lorentz factor of the electron range at least 300 - 90000 with a single power-law energy distribution (Tashiro et al. 2009).

\section{Observations and results}

The Suzaku mapping observations were conducted five times from 2007 to 2009, with a total exposure of $\sim 300 \mathrm{ks}$. The X-ray Imaging Spectrometer (XIS; Koyama et al. 2007) onboard Suzaku covers the energy range of $0.5-10 \mathrm{keV}$. The IC X-ray from the lobe is reported to dominate in energy range $2.0-5.5 \mathrm{keV}$ the surrounding thermal emission and backgrounds (Tashiro et al. 2009). We show the backgrounds subtracted XIS image in $2.0-5.5 \mathrm{keV}$ in Fig. 1a, which was smoothed with a two-dimensional Gaussian of $\sigma=2^{\prime}$. The obtained X-ray image shows the diffuse X-ray distribution associated to the radio lobe, but we see no significant trend along the jet axis. This supports that the lobe is dormant or inactive as suggested by Iyomoto et al. (1998) and Kim \& Fabbiano (2003). Considering these evidences of inactivity of jets, we assume a concentric energy distribution below. 

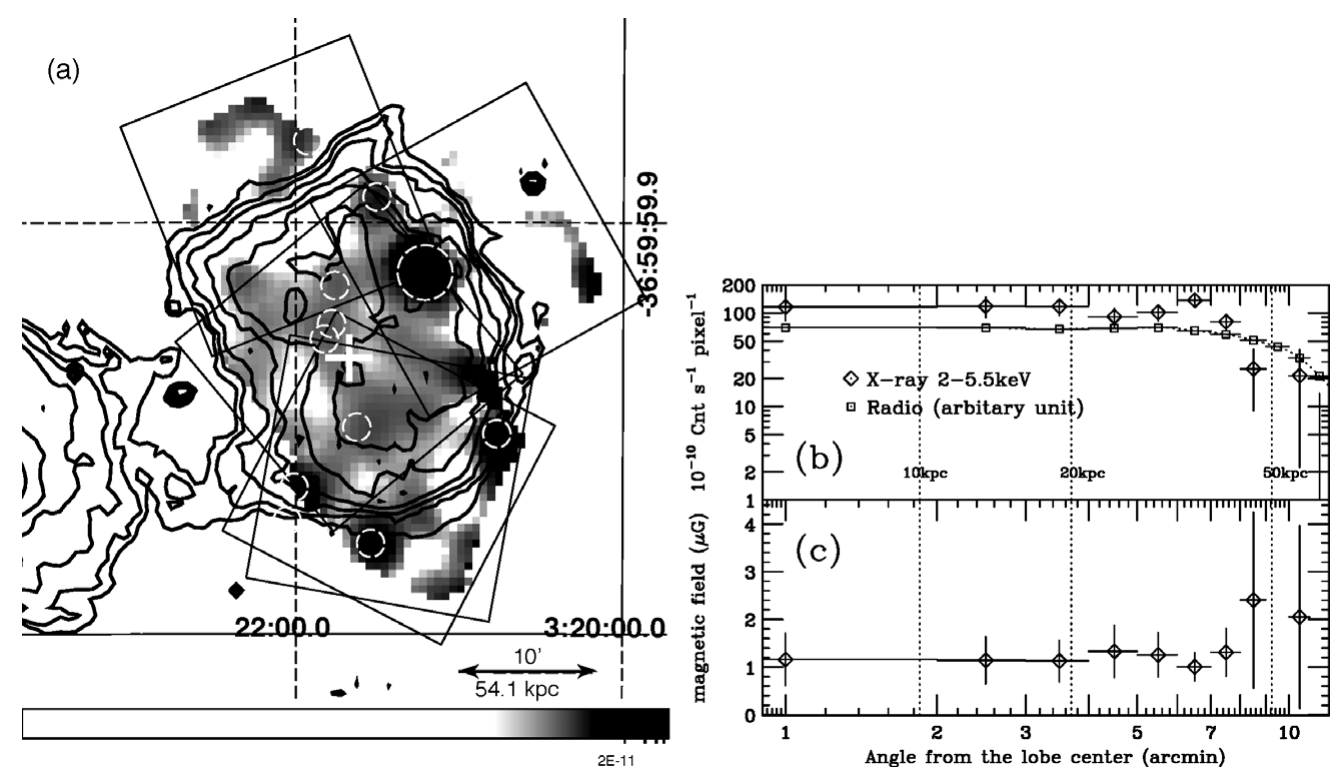

Figure 1. Panel (a) shows background subtracted XIS image in $2.0-5.5 \mathrm{keV}$. The $1.5 \mathrm{GHz}$ VLA contour image (Fomalont et al. 1989) is overlaid. The squares are the fields of view for XIS. The dashed white circles are subtracted contaminant sources. The cross white point is the center of the radial profile as shown in the regions of panel (b) and (c). Panel (b) shows the obtained X-ray count rate radial profile. The radio radial profile is overlaid. Panel (c) shows the derived distribution of $B$ from the ratio of $\mathrm{X}$-ray and radio flux.

The panel (b) of Fig. 1 shows the obtained X-ray radial profile. The $\mathrm{X}$-ray emission distributes up to $8^{\prime}$ from the center, and decreases rapidly in the outer region. The X-ray distribution is similar to, or smaller than that of radio emission. This indicates that $u_{e}$ distribution concentrates on the center of the lobe and is weak in the edge of lobe. By comparing the X-ray and radio fluxes in each radial region, the $B$ is found to exhibit relatively uniform $\sim 1 \mu \mathrm{G}$ in the lobe as shown in Fig. 1. This result may indicate that the particles loss their energy in the ambient B filling the expanding lobe.

\section{References}

Fomalont, E. B., Ebneter, K. A., van Breugel, W. J. M., \& Ekers, R. D. 1989, ApJL, 346, L17 Isobe, N., Tashiro, M., Makishima, K., Iyomoto, N., Suzuki, M., Murakami, M. M., Mori, M., \& Abe, K. 2002, ApJL, 580, L111

Iyomoto, N., Makishima, K., Tashiro, M., Inoue, S., Kaneda, H., Matsumoto, Y., \& Mizuno, T. 1998, ApJL, 503, L31

Kim, D.-W. \& Fabbiano, G. 2003, ApJL, 586, 826

Koyama, K., et al. 2007, PASJ, 59, 23

Tashiro, M. S., Isobe, N., Seta, H., Matsuta, K., \& Yaji, Y. 2009, PASJ, 61, 327 